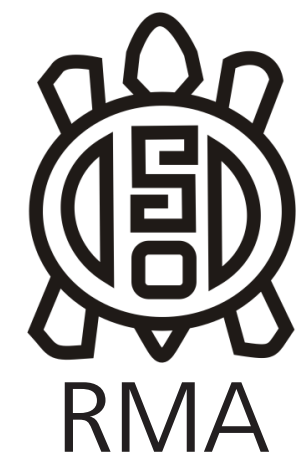

Arqueología

\title{
Habitando espacios vacíos. Cuerpos, paisajes y ontologías en el poblamiento inicial del centro de Argentina
}

\author{
Dwelling empty spaces. Bodies, landscapes and ontologies in the early \\ peopling of central Argentina \\ Andrés Laguens*, Benjamin Alberti** \\ * Instituto de Antropología de Córdoba (IDACOR, Universidad Nacional de \\ Córdoba - CONICET, Argentina). E-mail: andreslaguens@gmail.com \\ ** Framingham State University, USA. E-mail: balberti@framingham.edu
}

\begin{abstract}
Resumen
Los modelos de poblamiento inicial de territorios desconocidos implícitamente ponen en juego ontologías del cuerpo y del espacio o la naturaleza, con dos ideas fuertes: la de espacios vacíos de humanos y la de un medio desconocido, una 'terra ignota'. Se trata siempre de un encuentro entre grupos humanos y un espacio a descubrir, a experienciar, y en cuyo recorrido las poblaciones van relacionándose activamente con materialidades particulares y construyendo sus paisajes. Nuevas indagaciones nos llevan a cuestionar esta visión y la de una arqueología del paisaje que no considere la particularidad ontológica de la idea de cuerpo. Nos remitimos a un caso de estudio en San Luis, Argentina, y veremos qué sucede cuando el mismo es visto desde una ontología particular situada - en este caso el perspectivismo sudamericano. Entendemos que poblar 'terra ignota' desde un cuerpo y una ontología perspectivista no es crear paisajes, sino habitar un espacio de interrelaciones que, si bien se va definiendo y manteniendo activa y constantemente a través de relaciones, prácticas y experiencias entre sujetos, humanos y no humanos, en definitiva es un mundo de interrelaciones pre-constituidas, a partir de la propia ontología de los sujetos en cuestión.
\end{abstract}

Palabras claves: Ontología; Perspectivismo; Poblamiento; Paisaje; Cuerpo.

\begin{abstract}
Models of initial peopling of unknown territories implicitly bring into play ontologies of the body and of space or nature, with two strong ideas: that of empty spaces of humans and of an unknown milieu, a "terra ignota". It is always about an encounter between human groups and a space to be discovered, to be experienced. In that path people actively interact with particular materials while building their landscapes. New inquiries lead us to question this vision and that of a landscape archaeology that does not consider the ontological particularity of the idea of the body. We refer to a case study in San Luis, Argentina, and we will see what happens when the same is seen from a particular situated ontology - South American perspectivism. We understand that peopling 'terra ignota' from a perspectivist body and ontology is not to create landscapes but to dwell a space of interrelations that, although defined as well as actively and constantly maintained through relations, practices and experiences between human and non-human subjects; ultimately it is a world of pre-constituted interrelations, based on the own ontology of the subjects in question.
\end{abstract}

Key words: Ontology; Perspectivism; Peopling; Landscape; Body.

Quizás entre los temas más comunes en los que la constitución del paisaje adquiere un rol fundamental es en los modelos de movilidad de poblaciones humanas, tales como migraciones, desplazamientos, circuitos de intercambio, movilidad estacional. Entre ellos, los modelos de poblamiento inicial de territorios desconocidos -tal como el caso del continente americano en el límite Pleistoceno-Holoceno- son particularmente interesantes, en tanto implícitamente ponen en juego ontologías del cuerpo y del espacio o la naturaleza, usualmente sin mucha consideración adicional.

Entre esas concepciones y categorías, encontramos que la idea de paisaje, tal como está siendo entendida hoy en la arqueología desde la mayoría de las distintas posiciones teóricas vigentes, se ve altamente desafiada cuando intentamos abordar el paisaje desde una perspectiva relacional que tenga en cuenta otras 
ontologías no occidentales. Un aspecto importante es que las relaciones de los humanos con el paisaje no pueden ser pensadas sin considerar la idea de cuerpo, que implícita o explícitamente es puesta en juego no sólo en la relación en sí, sino en cada punto de vista o teoría. Podemos pensar a los humanos o a los cuerpos ya sea como sujetos de la experiencia, actantes en una red de relaciones, seres en interrelaciones vivas con otros seres del mundo, o de otras varias formas teóricas actuales. Pero, además, aceptando y sabiendo que hay otras ontologías - a las que podemos acercarnos desde la arqueología - sostenemos que tampoco podemos seguir haciendo una arqueología del paisaje que no considere las ideas de cuerpo y de persona, propias de la ontología de aquellos grupos con los que trabajamos.

Como ejemplo de ello nos remitiremos a un caso de estudio trabajado por uno de nosotros, el poblamiento inicial de Sudamérica (Laguens 2009, 2012). Veremos qué sucede cuando lo planteamos desde una concepción generalizada y occidental de sujeto y cuerpo, y cuando lo mismo es visto desde una ontología particular donde los sujetos no son sólo los humanos, sino también otros no humanos que son parte del paisaje.

Por ello, la propuesta que queremos presentar es doble. Por un lado, pensar los cuerpos/personas desde ontologías situadas para luego, desde allí, considerar los límites o alcances de las ideas relacionales de paisaje de la arqueología. Por otro, queremos plantear como otra meta poner en práctica el "giro ontológico" desde problemas y casos particulares, y así invitar a más reflexión sobre el tema de un arqueología que considere las ontologías (Alberti y Marshall 2009). En este último sentido, podemos entender a este estudio como "experimental", en tanto intentamos reflexionar y pensar las relaciones de los humanos con otras entidades de su entorno conjuntamente con otra [u otras] teorías nativas de la realidad, a la par de la nuestra. Organizamos el trabajo con una primera sección donde presentamos ideas sobre el poblamiento inicial de nuevos espacios, con la presentación sucinta de un caso en estudio que inspiró la búsqueda de otras interpretaciones; y una segunda sección, pensada más a modo de un ensayo sobre las posibilidades del perspectivismo amazónico como marco teórico interpretativo, en un ejercicio para destacar la importancia de incluir la visión ontológica en las interpretaciones sobre el poblamiento.

\section{Ideas de poblamiento inicial}

Cuando se piensa el poblamiento inicial de distintos continentes, usualmente hay dos imágenes muy fuertes asociadas con ello: por un lado, la de espacios vacíos de humanos y, por otro, la de un medio desconocido, una terra ignota (por ej. Borrero 1989-90, 2005; Kelly 2003; Meltzer 2013; Miotti y Saleme 2003; Webb y Rindos 1997, entre otros, y varios artículos en Rockman y Steele 2003; ver éste). Es un espacio sin otras gentes, sin información, sobre el que no habría experiencia, sin vínculos establecidos entre la gente y el ambiente. Se trata siempre de un encuentro entre grupos humanos y un espacio a descubrir, a experienciar, y en cuyo recorrido las poblaciones van relacionándose activamente con materialidades particulares de distinta escala, aunque aún sin significados. En este sentido, el poblamiento inicial resulta en una especie de génesis primigenia del paisaje - tanto como concepto como entidad particular - y donde usualmente dichas materialidades - lo dado o la naturaleza - juegan un rol fundamental (y activo) en la interacción. Se crean así nuevos lugares y se entablaban nuevas relaciones durables - económicas, cognitivas, adaptativas en general, o aún míticas - donde antes no existían. Es un proceso de ir transformando lo desconocido en conocido, lo indeterminado en determinado. De hecho, poblar terra ignota es ir transformando el espacio en paisaje.

Hemos argumentado con anterioridad (Laguens 2009) que los grupos de cazadores-recolectores americanos en su trayectoria de desplazamiento llevaban consigo a toda su sociedad, su pasado, sumado a redes de relaciones (políticas, sociales, familiares, económicas y demás), más series de disposiciones durables incorporadas o habitus particulares. Junto a ello, también portaban representaciones sociales, formas de entender y relacionarse con la naturaleza, categorías, ontologías propias, etc.; en breve, un todo complejo que iba haciendo del espacio, a donde sea que fuera, su "mundo" (por cierto, tal como cualquiera de los humanos, sean cazadores o no). En cada acto, un individuo o los individuos crean y re-crean esos mundos. Es un ir construyendo permanentemente lugares, que se van definiendo y cimentando como tales a través de las prácticas.

Y aunque el espacio nuevo sea "desconocido", siempre va a ser percibido y vivido de cierta forma, con capacidades para entender a priori ciertas claves del mismo, comprender las experiencias, sensaciones, hacer balances, tomar decisiones, etc. y por ende, relacionarse de ciertas formas con las cosas. A partir de allí se irán posibilitando la creación y recreación de interrelaciones particulares, tanto individuales como colectivas, situadas social e históricamente. Esos eventos de recreación, en cuanto prácticas, actualizan conocimientos tradicionales compartidos, y en tanto formas de hacer socialmente incorporadas y heredadas, se remontan a una larga duración. Son modos de entender el mundo y ontologías que lo ordenan, y que los individuos portan consigo en el transitar y poblar de nuevos espacios. Es una memoria social incorporada, que no sólo se porta en los cuerpos y la mente, sino también en las cosas, en los objetos, a la manera propuesta por Leroi-Gourhan (1965). Tal como dice Bender (2001), los paisajes son una red de relaciones, densa y compleja, entre gente, 
cosas y lugares, que no pueden ser separados de la experiencia humana, y que no pueden ser reducidos como puramente visuales; por el contrario, "son parte de un mundo de movimientos, relaciones, memorias e historias" (op.cit.: 76).

Hay que considerar que también el espacio será activo, y no será una dimensión externa, en oposición, con la cual se interactúa o a la que hay que ajustarse, como en general sucede en las discusiones relativamente recientes sobre el poblamiento (por ej., varios artículos en Rockman y Steele 2003). Ya de por sí, la propia materialidad del espacio tiene capacidad de acción, incidencia o agencia, sobre los individuos. Las cosas del entorno brindan para la gente distintas posibilidades o affordances (en términos de Gibson 1986; ver también Ingold 2007) que serán percibidas de acuerdo a sus disposiciones sociales o habitus.

A su vez, en el mismo proceso de construcción del paisaje hay un reconocimiento de categorías propias en la materialidad del espacio. No es necesario conocer un río particular para saber que se trata de un río, o llegar hasta una montaña para saber que habrá piedras, más allá de las categorías o significados en juego. Pero sí debo experimentar con las piedras si no conozco la materia prima. Es un reconocimiento basado en una forma de entender el mundo que, a la vez que construye ese mundo, lo incorpora en la experiencia y a lo largo del tiempo, tanto del vivir cotidiano como del largo plazo de la ocupación. Paisaje y personas son mutuamente construidos en su interrelación.

Podemos entender así que habitar el espacio es ir definiéndolo activamente y constantemente a través de relaciones, prácticas y experiencias entre humanos y no humanos. La definición de paisajes en el poblamiento puede ser vista como una re-creación de relaciones al habitarlo. En cierta medida, la terra ignota no resultaría tanta o muy desconocida por mucho tiempo.

\section{El poblamiento inicial visto desde Estancia La Suiza}

El caso particular que nos lleva a estas consideraciones es la arqueología del sector austral de las Sierras Pampeanas, donde hallamos un conjunto de sitios que por su conjunto artefactual y las formas de uso del espacio local son asimilables al poblamiento inicial de parte de Sudamérica. Se trata de la localidad arqueológica de La Suiza, en la provincia de San Luis, donde hallamos un sistema de sitios de cantera-taller y lugares transitorios y de uso reiterado de manufactura de artefactos líticos, caracterizados por ciertas etapas de tecnologías de reducción bifacial y la producción de puntas de proyectil de morfología cola de pescado (Laguens et al. 2007a, 2009; Sario 2010). No disponemos aún de fechados radiocarbónicos, aunque las características de los contextos tecnológicos y las puntas "cola de pescado",

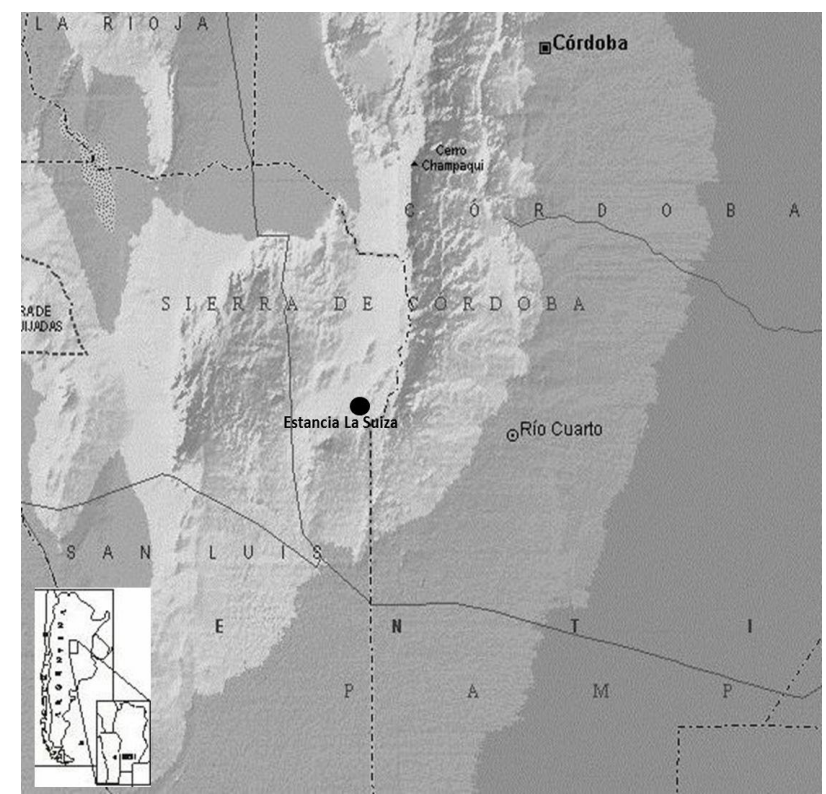

Figura 1: Ubicación relativa del sitio Estancia La Suiza

Figure 1: Relative location of the Estancia La Suiza site

permiten aventurar su correspondencia cronológica con el final del Pleistoceno.

La localidad incluye alrededor de una decena de sitios con propiedades similares, en un radio de aproximadamente $2 \mathrm{~km}$ alrededor de un arroyo temporario y su confluencia con un río en el piedemonte occidental de las Sierras de Comenchigones $\left(32^{\circ} 56^{\prime}\right.$ de latitud Sur y $65^{\circ} 07^{\prime}$ de longitud Oeste, y a 942 msnmm, Figura 1). Se trata de sitios al aire libre y también de sitios definidos a partir de artefactos en los perfiles de barrancas de cauces temporarios. Es un área rica en recursos minerales, con afloramientos de chert y cuarzo (Laguens et al. 2007a).

De particular relevancia son los hallazgos en el sitio Estancia La Suiza 1 (ELS1, en adelante), donde se encontraron en superficie dos puntas "cola de pescado" o tipo Fell 1, así como los sitios ELS 2 y ELS 4, dos canteras de chert, ópalo y cuarzo, utilizadas como fuente de aprovisionamiento en dicha localidad; y el sitio ELS 3, donde excavaciones estratigráficas han permitido avanzar en la comprensión del conjunto tecnológico lítico de estas ocupaciones (Laguens et al. 2007a y b, Sario 2010).

Los restos líticos obtenidos de prospecciones sistemáticas en ELS 1 incluyen 2 fragmentos de puntas cola de pescado (Figura 2) e instrumentos como raspadores, manos, cuchillos, unifaces, bifaces, preformas, lascas retocadas y fragmentos de núcleos (Figura 3). Aunque el $70 \%$ de los artefactos descartados están fragmentados, la mayoría de las lascas corresponden a los primeros estadios de la manufactura, pero también se encuentran lascas de reducción de bifaces (ver Sario y Pautassi 2010 y Sario 2007). 


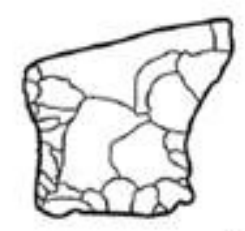

A
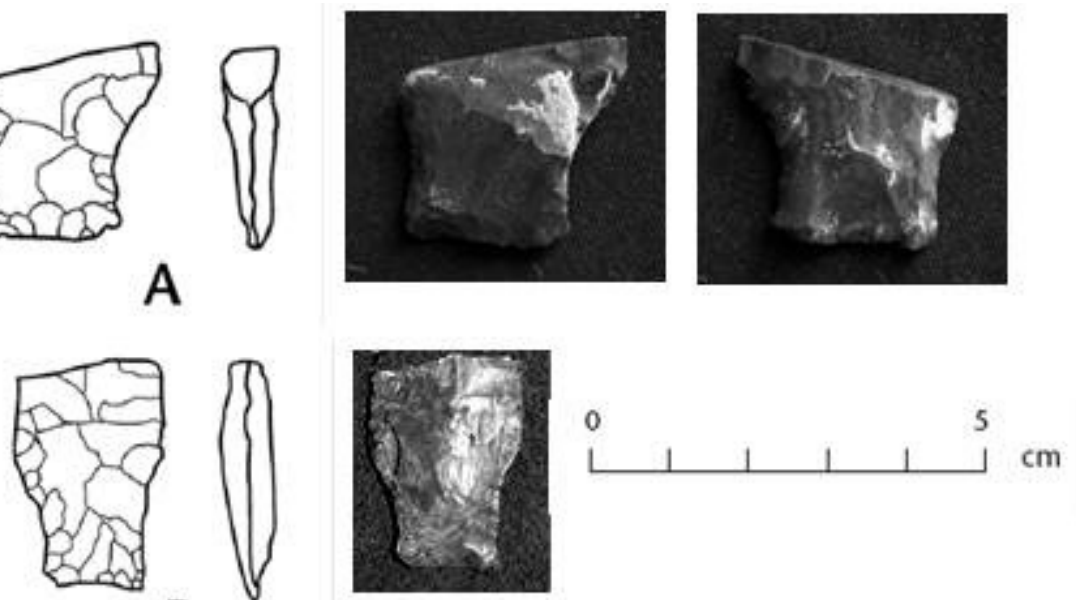

B

Figura 2: Fragmentos de puntas colas de pescado halladas en el sitio Estancia La Suiza. (A): procedente de ELS 1/b; (B): procedente del ELS1 1/a

Figure 2: Fragments of fishtail projectile points found at the Estancia La Suiza site. (A): from ELS 1/b; (B): from ELS1 1/a

A $1,2 \mathrm{~km}$ al Oeste de este sitio se halló una cantera arqueológica (Sitio ELS 2), de una variedad de rocas silíceas de excelente calidad para la talla, entre ellas: chert, vulcanitas, ópalo y calcedonia y, en menor cantidad, otros materiales silíceos, con una concentración de materiales superficiales que incluyen núcleos, instrumentos y lascas.

El sitio Estancia La Suiza 3 (ELS 3) se halla a unos $180 \mathrm{~m}$ al Noroeste del sitio ELS 1, donde se realizó una excavación extensa, y se identificaron 17 unidades estratigráficas (UE 1 a UE 17), desde la superficie actual hasta 1,40 m de

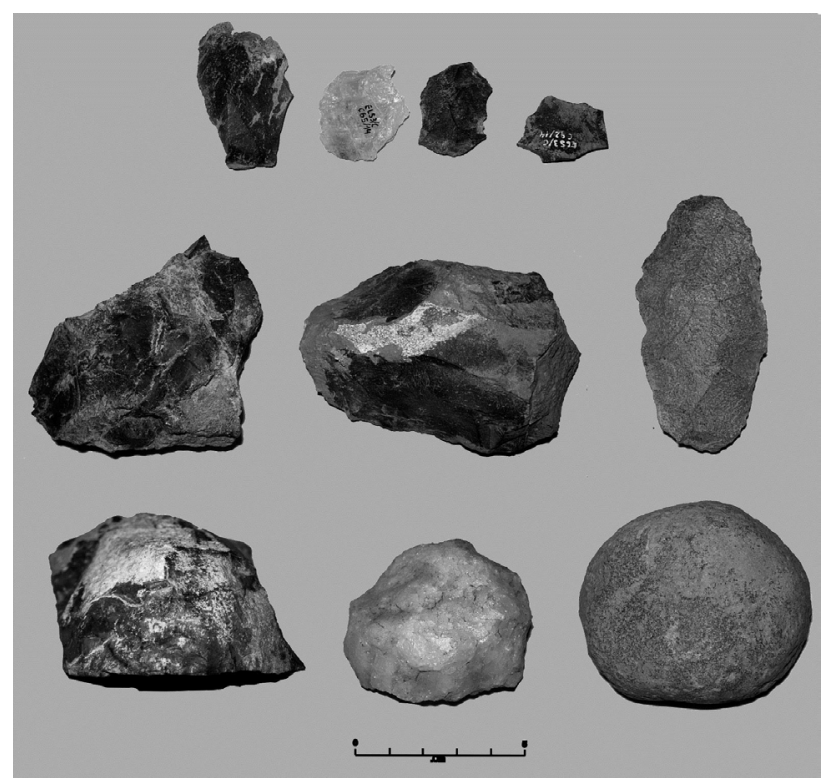

Figura 3: Artefactos líticos de ELS 3, Unidad Estratigráfica 14-16

Figure 3: Lithic Artifacts of ELS 3, Stratigraphic Unit 14-16 menor proporción a instrumentos formatizados, confeccionados mayoritariamente sobre el chert local (Laguens et al. 2007a, Sario 2007). Este componente se hallaría vinculado tecnológicamente con las puntas cola de pescado del sitio ELS 1. El material del Componente II, corresponde en su mayoría a desechos de talla y en mínima proporción a instrumentos (4 objetos), confeccionados sobre el mismo chert, con algunos elementos en cuarzo.

Estos hallazgos plantearon varios puntos interesantes: por un lado, estarían confirmando la presencia en la zona de poblaciones vinculadas con el poblamiento continental inicial; por otro, desde el punto de vista de la tecnología, dadas sus similitudes en morfología y la lógica de su producción, estarían vinculadas con la Pampa y Patagonia, a la par que con los procesos poblacionales de dichas regiones.

\section{La Suiza en el contexto más amplio del poblamiento regional}

En particular, nuestro esquema básico sobre el poblamiento local sostiene que en el límite PleistocenoHoloceno, se comienza a producir el ingreso de poblaciones humanas al centro de Argentina desde el Este de la región, a diferencia de lo que se venía pensando tradicionalmente en cuanto a un poblamiento de origen andino desde el Noroeste. Este ingreso inicial se habría producido a lo largo de importantes ríos de las Ilanuras pampeanas pertenecientes a la cuenca del Paraná-Río de la Plata que, actuando como corredores ambientales, pudieron funcionar como vías de menor costo para el desplazamiento, a la par de asegurar recursos. Se habría tratado de una opción migratoria a partir de la fisión de grupos que, continuando una 
ruta principal de desplazamiento en dirección nortesur, habrían de terminar asentándose en la Pampa y Patagonia (Laguens et al. 2003; Laguens 2008).

Una serie de dataciones radiocarbónicas para la región ayudan a sostener su poblamiento temprano. En el sitio El Alto 3, un abrigo a 2.200 msnmm en la Pampa de Achala, Córdoba, se hallaron lascas y artefactos en cuarzo, con dataciones de $11.010 \pm 80$ años C14 AP (LP-1506), $9.790 \pm 80$ años C14 AP (LP-1420) y 9.371 \pm 51 años C14 AP (AA94987) (Rivero y Berberián 2011, Rivero y Roldán 2005). En otro sitio en las sierras, la Gruta de Candonga (donde en los '40 se encontró una posible asociación de fauna extinguida con restos humanos), se obtuvo más recientemente un fechado sobre los huesos humanos que brindó $10.450 \pm 50$ años C14 AP (SRLA-1062) (Cornero y Neves 2011). Si bien pocas, estas ocupaciones humanas estarían confirmando la hipótesis de presencia humana efectiva hacia fines del Pleistoceno en el Este de la región, antes que en el Oeste, donde hasta ahora los fechados más antiguos se remontan a 8000 años atrás en sitios con tecnología de puntas lanceoladas (v.gr. Ayampitín). En el sitio El Alto 3 la tecnología de puntas lanceoladas se halla superpuesta estratigráficamente en ocupaciones posteriores que siguen a los niveles de los fechados del límite Pleistoceno-Holoceno.

En cuanto a la relación con otros grupos y con los procesos de poblamiento de las llanuras del Este y Sur de Argentina, a través de estudios bioantropológicos de morfología craneal (Fabra 2014; Fabra et al. 2005; Laguens et al. 2007) y ADN antiguo (Nores y Demarchi 2011, Nores et al. 2011), se pudo determinar que los habitantes de estas sierras muestran similitudes morfológicas más cercanas con las poblaciones de Patagonia y Tierra del Fuego que con los de otras regiones aledañas.
Enlazando estos hallazgos con el poblamiento de parte del continente sudamericano en el límite PleistocenoHoloceno, hemos planteado que este proceso histórico se asemeja más al de una diáspora que al de una migración, en cuanto se trata de poblaciones que comparten un origen en común y que portan consigo una historia y un modo de entender y relacionarse con el mundo (Laguens 2009). Esto toma sustento en el hecho conocido que, más allá de paisajes o contextos ambientales distintos, en muchos puntos dispersos en el continente y extremadamente alejados entre sí, en diferentes biomas, las mismas prácticas son reproducidas independientemente. Iguales o similares morfologías de artefactos - como las puntas "cola de pescado" - son halladas a través de casi todo el sub-continente, más o menos contemporáneamente entre los 11.000 y 10.000 años A.P. (i.e. las sabanas de Colombia; en los Andes en Ecuador, Perú Chile y Argentina - en la Puna, pre-puna salteña, Mendoza y Neuquén - así como en las planicies uruguayas y en las sierras pampeanas, la pampa húmeda y la Patagonia en Argentina) (Patané Aráoz y Nami 2014; ver la Fig. 21.1 en Flegenheimer et al., 2014: 360; Politis et al. 2004). Junto con ellas son reiterados los hallazgos de las mismas técnicas de talla, así como sistemas de sitios de cantera-taller, lugares transitorios y de uso redundante para la manufactura de artefactos líticos; en particular, éstos últimos caracterizados por ciertas etapas de tecnologías de reducción bifacial y la producción de las puntas de proyectil de dicha morfología "cola de pescado", las que luego son halladas en otros contextos. Hay así una reiteración de acciones breves, puntualmente localizadas en espacios acotados y seleccionados, donde una y otra vez se reproducen a lo largo del tiempo las mismas prácticas, las mismas secuencias de eventos, registradas de manera similar en distintos sitios en varias y diferentes regiones, con formas análogas de uso del espacio, con similares lógicas tecnológicas así como modos de vida análogos, en general. En particular,

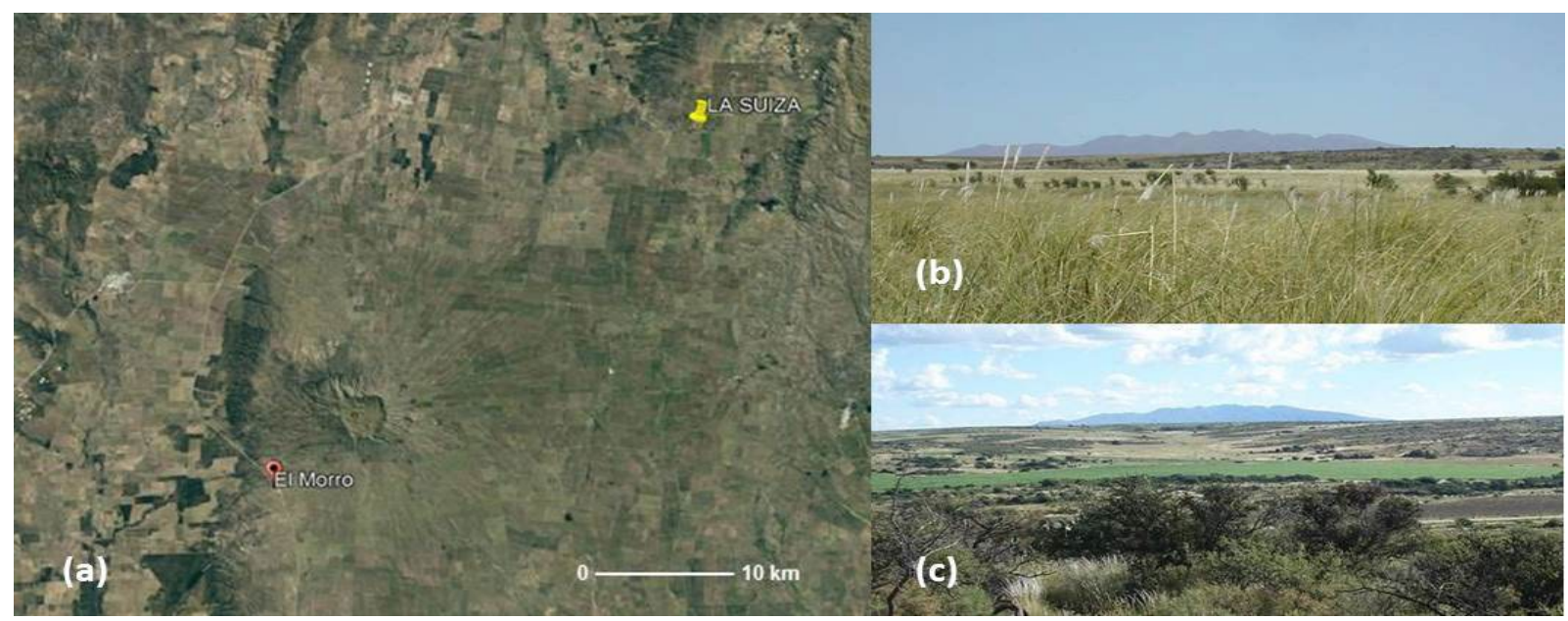

Figura 4: Ubicación relativa del sitio La Suiza en relación al volcán apagado Cerro El Morro. (a) imagen satelital (basada en Google Earth); (b) Vista del cerro El Moro desde el sitio; (c) vista del Cerro El Morro desde la cantera

Figure 4: Location of the site La Suiza in relation to the extinct volcano El Morro. (a) satellite image (based on Google Earth); (b) View of Cerro El Moro from the site; (c) view of Cerro El Morro from the quarry. 
es llamativa la elección de espacios para habitar en proximidades de formas naturales conspicuas en el paisaje, como volcanes, mesetas, cerros destacados en planicies, o alguna formación geológica, que actuaron como atractores o nodos físicos en las redes sociales (ver Flegenheimer et al. 2013), tal como sucede en La Suiza en relación al cerro El Morro, un volcán apagado a $32 \mathrm{~km}$ de distancia, cuya presencia conspicua es visibilizada permanentemente desde el sitio y toda la zona aledaña (Figura 4). Pensamos que estos eventos y contextos locales pueden entenderse como formas de reproducción y objetificación de estructuras compartidas de manera amplia por parte de gente viviendo sus vidas cotidianamente, en una escala geográfica mucho más amplia que lo local, casi continental.

En definitiva, todo apunta a pensar que se trataba de una misma gente, de un grupo social disperso común, con lógicas similares, con concepciones similares del mundo. Se refuerza así la idea de un origen en común en esta etapa inicial del poblamiento con las características de una diáspora, quizás con un sentido de identificación o pertenencia compartido, materializado cotidianamente en los objetos y el espacio (Laguens 1999; ver Bradley y Collins 2013, quienes realizan una argumentación con cierta similitud para Clovis en América del Norte desde la perspectiva de movimientos sociales de revitalización, en los cuales hay involucrados procesos de reformulación cultural, adaptación, comunicación, rutinización, entre otros, de los cuales resultan nuevos estados).

Cualesquiera sean las explicaciones, podemos pensar que en diferentes lados entraron en juego de manera similar disposiciones durables incorporadas o habitus que permitieron que, a través de prácticas semejantes, los cazadores-recolectores tempranos fueran haciendo en los paisajes sus mundos particulares, más allá de las marcadas variaciones espaciales y ambientales.

\section{Otras ontologías, otros paisajes}

Ahora bien, toda la descripción anterior no deja de ser un modelo de poblamiento - del desplazamiento de humanos en un espacio natural - que puede ser potencialmente válido para cualquier tiempo y lugar del planeta, no sólo el continente americano, y planteado a partir de una ontología europea occidental. Y si bien este modelo considera humanos situados social e históricamente, sin embargo asume un espacio pre-conceptual real, objetivo, sujeto a interpretaciones relativas de acuerdo a cada grupo social, y concomitantemente, a construcciones o recreaciones particulares. En breve, espacios pasibles de cobijar distintas formas sociales de representar sobre él sus propios mundos, los que así se convertirán en paisajes (culturales).

Sin embargo, y si bien declaramos la ubicuidad socio- histórica de estas formas de relacionarse con los espacios - co-generando múltiples y distintos "paisajes" -, consideramos que aun así seguimos pensando en potenciales sujetos homogéneos, en tanto humanos genéricos morando espacios diversos, subordinados a idénticos procesos de relación con y de construcción de paisajes, con una idea de cuerpo o de sujeto noespecífica ontológicamente.

El tema puesto en relieve es la manera en que gente con una ontología particular, como un modo de entender el mundo, y una memoria social en común, transita y puebla nuevos espacios, re-creando así sus mundos, más allá de adaptaciones y variaciones particulares.

Queremos ir un paso más allá. Intentamos desafiar este esquema de performatividad de las prácticas rutinarias en un espacio vacío y desconocido, enriqueciéndolo con otra/s idea/s de sujeto, de cuerpo y de mundo; básicamente, experimentando con otra ontología como teoría.

¿Qué pasa cuando sujetos de otra/s ontología/s que entienden de otra manera a los individuos y al mundo se enfrentan con un espacio desconocido y vacío, con terra ignota? En particular, nos preguntamos, ¿Cómo podemos pensar estos mismos datos y este mismo modelo de poblamiento inicial por parte de sujetos perspectivistas?

Elegimos trabajar con el perspectivismo amazónico como una ontología sudamericana de base amplia (aunque también propia de otros lugares del mundo, como América del Norte, Siberia, Mongolia, Vietnam e Indonesia; ver Halowell 1960; Howell 1989, Pedersen 2007; Willerslev 2007) y como tal, consideramos que puede ser de validez para nuestro caso de estudio, porque desafía deliberadamente supuestos ontológicos que comienzan desde el cuerpo.

Nos interesa destacar que esto no es analogía etnográfica: no esperamos encontrar cuerpos y paisajes amazónicos, sean las que sean. Estamos desarrollando una forma alternativa de mirar a la idea de paisaje que concuerda mejor con lo que podríamos encontrar arqueológicamente trabajando a través de las teorías desarrolladas sobre la base de otras ontologías (véase Alberti y Marshall 2009). Nuestro argumento es que no podemos llegar a estos paisajes arqueológicos a partir de las teorías tradicionales, ya que implican un tipo específico de conceptualización del cuerpo que cercena una serie de alternativas ontológicas. La propia idea de paisaje es un artefacto o efecto del concepto occidental de cuerpo, ya sea como plataforma de observación neutra, o ente sensible a través del cual formamos relaciones con paisajes particulares y los llevamos a su existencia. Podríamos haber elegido otra ontología, pero creemos que el perspectivismo aporta una teoría nativa 
del mundo mucho más situada para nuestro caso que otra de cualquier otro lado, o de base universal, como el animismo en general (ver Troncoso 2014 para una crítica del uso generalizado del animismo en arqueología y una posición similar a la sostenida aquí).

\section{Perspectivismo}

El perspectivismo amerindio es una concepción indígena según la cual el mundo está poblado por diferentes entidades, agentes o personas (tales como ciertos animales, espíritus, objetos, fenómenos de la naturaleza, los artefactos y las plantas), todos los cuales son considerados sujetos, y que, como tales, ven el mundo de la misma manera que lo hacen los humanos. El carácter distintivo reside en sus cuerpos, que son el hogar de la subjetividad y de su punto de vista o perspectiva. En consecuencia, los diferentes tipos de seres no ven las mismas cosas, sino que ven las cosas específicas a partir de la perspectiva de su propio mundo, que es cultural, humano. Por ejemplo, los seres humanos ven a los pecaríes como animales, pero los pecaríes se ven a sí mismos como personas y ven a los humanos como presas o enemigos. El modo de ver, por lo tanto, es siempre el mismo - es un acto cultural. Lo que es diferente es lo que se ve. La cultura es una, lo que cambia es el mundo, o la naturaleza. Esta es la base de lo que Viveiros de Castro llama "multinaturalismo", en oposición al multiculturalismo.

De acuerdo con el perspectivismo, la humanidad indiferenciada es la condición universal y original de todas las entidades, humanos y no humanos (Viveiros de Castro 1998, 2004a; Lima 1999, 2000). Cualquier entidad puede tener un alma humana y ser una persona, y hay una subjetividad semejante a la humana que es compartida de manera generalizada, más allá de las particularidades de los cuerpos o la forma externa de las cosas (Viveiros de Castro 2004b).

\section{Perspectivismo, cuerpos y paisajes}

Partimos de una arqueología del paisaje que reconoce la reciprocidad teórica de los conceptos de cuerpo y paisaje y las ideas de cuerpos de etnografías amazónicas, en tanto ilustran esa relación estrecha, así como proporcionan una idea alternativa del paisaje que se puede utilizar en arqueología. En lugar de una relación entre un cuerpo genérico (sin embargo socialmente modificada) y una variedad de paisajes de tipo contenedor, vemos paisaje y cuerpo constituidos por múltiples seres a través de sus relaciones. Así, decidimos tomar el habitar los espacios vacíos en tanto una dimensión inherentemente relacional donde los cuerpos humanos y no humanos participan activamente.

Los cuerpos son de importancia central para el perspectivismo. De acuerdo con Viveiros de Castro, los cuerpos no son tanto el componente biológico sino que son "conjuntos de afectos", una serie de capacidades y formas de responder que los cuerpos comparten con cuerpos similares. Todos los seres comparten la misma capacidad de ver y conocer el mundo, como hemos visto. Lo que los diferencia son sus cuerpos - cuerpos que no son objetivos referentes estables, sino que deben ser trabajados y capacitados para producir el "conjunto de afectos" correcto que asegure que van a hacer y ver de la misma manera que sus parientes o miembros de la especie.

Las perspectivas están situadas en los cuerpos. Los cuerpos son lo que diferencian a los sujetos $y$, en última instancia, los mundos que ocupan. De manera importante, las relaciones son claves en la constitución de los sujetos y sus mundos. Como señala Vilaça (2009, 136), el perspectivismo sostiene que "no hay una percepción pura anterior a las interacciones" entre los sujetos y los objetos; esto es, diferentes conjuntos de relaciones no producen diferentes objetivaciones de un único mundo objeto, sino que, "diferentes constituciones corporales del sujeto" $y$, por tanto, diferentes mundos.

Por ejemplo, a medida que exploramos la literatura sobre los cuerpos y el espacio de la Amazonía, vemos que en lugar de un espacio abierto de encuentro, las personas viven en mundos constituidos por múltiples subjetividades, u otras personas. Si extendemos estas relaciones para incluir formas del terreno, las plantas, los ríos, las estrellas, y demás, tenemos un mundo que se compone de interacciones entre seres - es decir, una sociedad de principio a fin. Y esto no es solo propio del perspectivismo: lo mismo es válido para los Andes, donde para muchas sociedades el mundo está poblado de múltiples subjetividades potenciales, incluyendo piedras, montañas, ríos, el cielo, los animales, antepasados, objetos y muchas otras entidades animadas. Términos tales como Camay (Salomon 1991), Sami (Arnold y Hastorf 2008), wak'a (Allen 2015; Bray 2015) son algunos de los conceptos que sintetizan un mundo que difumina los límites entre la humanidad, la materialidad y la subjetividad.

El entendimiento perspectivista de los cuerpos lleva a la pregunta de qué sucede cuando se desafía la ontología de los cuerpos (como máquinas de pensar-sentir, aunque si bien situadas, igualmente universales). Los cuerpos amazónicos parten de una premisa diferente que colapsa paisaje y cuerpo, porque el cuerpo es el punto de vista y el mundo está lleno de seres y de relaciones; no es un "mundo" inerte listo para ser esculpido por la acción humana. Los cuerpos son perspectivas; son los que hacen que un mundo aparezca de una manera determinada. No hay formas generalizadas en las que un cuerpo generalizado se distingue a sí mismo en su relación con un paisaje generalizado, por lo tanto 
haciéndolo específico. Nuestra posición es que esta noción de lo que es el cuerpo no funciona con una idea de "paisaje" como es entendido convencionalmente, sino que apunta hacia ecologías de múltiples yos (Kohn 2013) o subjetividades que constituyen de manera relacional todos los elementos de sus mundos.

El resultado es una perspectiva local del paisaje que incluye las ontologías y cosmologías locales en las relaciones ecológicas. Este tipo de relaciones, sostiene Mora para la Amazonía (Mora 2006, 15), son de hecho relaciones socio-ecológicas, ya que todas las especies se conciben como seres sociales, y cualquier espacio geográfico se define por y es lugar de relaciones sociales. El principio de organización que gobierna el universo es el de las relaciones sociales y de parentesco entre pares - de cualquier especie. A través de las relaciones sociales, los diferentes seres se auto-definen como seres humanos. Ellos habitan en mundos muy similares al nuestro en los que los seres humanos reales son vistos como otras criaturas del universo con las que mantienen relaciones de afinidad y reciprocidad. Por lo tanto, el espacio o la geografía están en permanente movimiento y transformación. Vivir en un paisaje así no es sólo mantener relaciones ecológicas y económicas con un espacio determinado, sino vivir en un mundo habitado por muchos otros sujetos habitantes del mundo.

El paisaje está poblado de entes vivos - no "animados" - y seres reflexivos con los que los humanos entran en relación. Desde esta perspectiva, vivir en un paisaje es habitar un espacio de relaciones con otros, los seres humanos y otros-que-humanos, con capacidades comunicativas (Kohn 2013). No se trata sólo de un paisaje: es un espacio que está totalmente compuesto por sujetos que interactúan.

\section{Poblando terra ignota}

Hasta aquí, vimos la naturaleza fabricada de los cuerpos en el perspectivismo y la noción extendida de lo humano a otras series de entes, y cómo las relaciones entre ellos - así como los modos de esas relaciones pueden producir distintas constituciones corporales de los sujetos, resultando en su permanente inestabilidad. Concomitantemente, no hay un otro fijo, definido e invariable. Asimismo, habrá tantas naturalezas como distintos puntos de vista, en tanto cada especie de ente que habita el mundo ve las mismas cosas de manera distinta, aunque siempre de manera cultural.

Pensemos ahora un cuerpo perspectivista, transitando un ambiente desconocido, por terra ignota.

Para un perspectivista el mundo está poblado de entes potencialmente humanos. No importa que el paisaje sea desconocido, igual el mundo es un mundo de sujetos y de relaciones, perspectivas y transformaciones. Allí los animales no serían solo animales, sino posibles personas, tanto como los árboles, los cerros u otras materialidades y entidades que pueblan ese espacio. Si bien es un entorno novedoso, desde su propia ontología se sabe que esos entes no humanos pueden transformarse en otros humanos (así como transformar a uno en otra especie), de acuerdo a cómo los sujetos se relacionen con ellos. Además, su experiencia y percepción de ese ambiente es una entre otras tantas posibles, considerando aquellas de los no humanos que también habitan el lugar. Las cosas pueden ser desconocidas en su exterioridad o en su forma, pero no en su modo de existencia. Luego, los modos de relacionarse con lo desconocido ya están definidos.

Desde esta ontología perspectivista, la potencial dificultad de transitar esos nuevos ambientes, desconocidos y distintos, no se resuelve (solo) por categorías previas proyectadas en el nuevo mundo, creando y re-creando paisajes traídos consigo. Sino que son los modos de relacionarse con lo otro lo que iría construyendo en todo caso un paisaje; modos ya definidos en la forma propia de entenderse a sí mismos, a los otros, y al mundo en general. Así transitar y ocupar un espacio ignoto puede resultar conflictivo, desafiante o estresante, no por lo desconocido, sino en todo caso por lo inestable de un mundo perspectivista, así como la del propio cuerpo en interrelación. No es la ansiedad de lo desconocido, sino la ansiedad de lo conocido. Y justamente en virtud de ello es que no podemos decir que sea un mundo desconocido. Puede ser materialmente distinto - y en ese aspecto desconocido - pero no en tanto mundo definido ontológicamente de una manera particular.

Consideremos también, que esa forma de entender al mundo y a los otros que lo habitan es cultural. Todos los entes (animados o inanimados) que lo pueblan, al ser o poder ser personas, tienen cultura; una única cultura: la humana (Viveiros de Castro 2004a). Entonces, allí donde todo lo material, lo animal, lo vegetal, conocido o desconocido, no son parte de una naturaleza sino que pueden ser otros humanos con sus propia culturas, las relaciones con el entorno ya no son relaciones ecológicas ni sólo relaciones perceptuales, experienciales, económicas o co-constitutivas, sino que resultan ser básicamente, y ante todo, relaciones entre sujetos. Potencialmente son relaciones humanas entre pares, relaciones más sociales que naturales. Y relaciones entre sujetos inestables, no fijas, cuya permanencia es constantemente cuidada y mantenida a través de las prácticas ¿Qué lugar queda para el paisaje desde esta perspectiva? ¿Hay un paisaje donde todo es transformable? ¿Qué lugar queda para las relaciones ecológicas o cómo debemos entenderlas cuando la relación depredadora es entre sujetos de distintas especies? ¿Cómo pensar el poblamiento donde no hay naturaleza? ¿Podemos hablar de exploración cuando se trata de modos de relación ya establecidos con lo 
desconocido? ¿Se coloniza un espacio o se habita, se convive?

Se podría entender este modelo como gente "llevando su paisaje" con ellos mismos. En lugar de imaginar el movimiento de personas de un lugar establecido a un nuevo paisaje que se impone sobre el grupo, podríamos pensar en los cazadores recolectores tempranos como llevando su paisaje - su punto de vista, anclado en sus cuerpos - con ellos cuando se mudaron por distintas tierras del continente. Esto implica un tipo de relación con los demás habitantes del mundo (sujetos, cuerpos humanos y no humanos) que es co-constitutiva de todos los elementos de ese mundo, humanos y otros-quehumanos. $Y$ el mundo mismo, en lugar de ser externo a ese proceso, en un sentido muy literal era el mismo, independientemente de que los "cuerpos" fueran externamente distintos (como otros árboles, montañas, las estrellas, y así sucesivamente). En consecuencia, la variación en los detalles de la cultura material dentro y entre lo que se ha llamado "colas de pescado" y sus contextos materiales locales es menos importante que las similitudes morfológicas. Es decir, las similitudes en las cosas indican relaciones subyacentes de un tipo similar (incluyendo relaciones prácticas con el mundo) (Alberti 2007).

Si retomamos el ejemplo, un aspecto llamativo de varios contextos arqueológicos particulares sudamericanos, es la frecuente vecindad y asociación directa de los sitios con elementos conspicuos del espacio (volcanes apagados, cerros aislados, mesetas destacadas en llanuras, por ejemplo). No es arriesgado pensar que estas formas geológicas hayan funcionado en algún sentido como elementos atractivos, quizás buscados intencionalmente, y pasibles de ser imbuidos de un valor o significado especial. No podemos afirmar que hayan sido entendidos como humanos (tal como los cerros o apus en los Andes), pero sí el rol central y participación en la organización del mundo de estos cazadores recolectores tempranos, quienes hacían uso de estos lugares reiteradamente, organizaban parte de su vida alrededor de los mismos, en constante interrelación. No dudamos que ontológicamente esos cerros hayan sido más que meros elementos del paisaje, más que una categoría clasificatoria y más que una cosa o materialidad particular cargada de significado. Tienen que haber sido parte activa y constitutiva de su mundo, y como tal, entes co-habitantes de los distintos paisajes de estos grupos tempranos en el continente. A través de situaciones y relaciones como éstas, podríamos decir que los cazadores recolectores fueron poblando a su manera un mundo, sus mundos.

\section{No-paisajes}

Si el mundo es un mundo de relaciones sociales entre especies humanas ocultas en distintas materialidades o cuerpos, donde toda materialidad es inestable, donde se experimentan más transformaciones que sensaciones, ¿podemos hablar de la existencia de paisajes como construcción o efecto de los modos de relación activa con el medio? ¿Hasta qué punto desde esta ontología relacional hay lugar para una co-construcción del paisaje, cuando la relación entre partes se da un mundo dado, con formas de relación pre-establecidas? ¿Se construye un paisaje donde los cuerpos - tanto de uno como de los otros no humanos - son múltiples, inestables y se van construyendo relacionalmente?

Creemos que no. No se trata de un espacio cartesiano de materialidades y entidades no humanas estables que puede ser experimentado - e inclusive animado - por los humanos a través de relaciones activas. El paisaje no resulta construido. Es más, quizás no haya lugar para pensar en paisajes. Lo que empezamos a ver aquí es un "paisaje" que en realidad es un "no-paisaje": un espacio imposible de fijar ni del cual tomar distancia.

\section{Consideraciones finales}

En síntesis, poblar terra ignota desde un cuerpo y una ontología perspectiva no es crear paisajes, ni un encuentro con lo desconocido, donde tampoco hay lugar para una agencia de las cosas en tanto meras cosas. Sino más bien se trata de habitar un espacio de interrelaciones que se va definiendo y manteniendo activa y constantemente a través de relaciones, prácticas y experiencias entre sujetos, humanos y no humanos, constitutivamente inestables.

Creemos, en suma, que hay que quitar la atención de las diferencias en el medio ambiente y sus materialidades. Lo que nos interesa es pensar en una forma de poblamiento y ajuste (adaptación) alternativa que se basa en una noción diferente del cuerpo y, por lo tanto, del paisaje. En el modelo tradicional, cuerpos y paisajes son distintos: los primeros tiene las herramientas para adaptarse a este último. Los seres humanos deben aprender a vencer, o, más amablemente, vivir con sus entornos. Cuando se parte de una ontología perspectivista - e inclusive otras - por el contrario, lo que importa es cómo se resuelve localmente y ontológicamente los cambios en el medio ambiente, como los que pueden suceder en los desplazamientos humanos por tierras desconocidas. La iniciativa es ir de la relación entre los seres humanos y el mundo a la relación entre muchos seres, incluyendo todos los elementos de lo que tradicionalmente se llama el paisaje. La forma de relacionarse y de constituir como humanos y/o no humanos entre la multitud de entidades del ambiente es lo que hizo posible la vida de estos grupos de cazadores recolectores en esos variados lugares que nosotros percibimos como diferentes.

Obviamente esto no quiere decir que planteemos que el poblamiento americano haya sido hecho por 
sujetos perspectivistas (más allá de las sugerentes similitudes entre el perspectivismo americano y el siberiano, así como la amplia distribución geográfica del perspectivismo en Sudamérica). No es lo importante ahora. Sólo intentamos experimentar con el hecho de cómo pensar a los sujetos ontológicamente situados puede cambiar la forma de entender este proceso y al paisaje, y así dar lugar a otros entendimientos, modelos, conceptos o aún explicaciones, si se quiere. A partir de ello nos preguntamos si al hablar de poblamiento (americano o más allá) podemos hacerlo sin considerar la ontología particular - así como la idea de cuerpo de los cazadores recolectores del límite pleistocenoholoceno que fueron habitando este continente.

La Paya, 10 Octubre 2016

\section{Bibliografía}

Alberti, Benjamin (2007) Destablizing meaning in anthropomorphic forms from Northwest Argentina. Journal of Iberian Archaeologv, 9/10: 209-29.

Alberti, Benjamin e Yvonne Marshall (2009) Animating archaeology: Local theories and conceptually openended methodologies. Cambridge Archaeological Journal 19(3), 344-56.

Allen, Catherine J. (2015) The Whole World Is Watching: New Perspectives on Andean Animism. In: The Archaeology of Wak'as: Explorations of the Sacred in the Pre-Columbian Andes, T. L. Bray (ed.) University Press of Colorado, Boulder. Pp. 23-46.

Arnold, Denisse Y. y Christine A. Hastorf (2008) Heads of State: icons, power, and Politics in the Ancient and Modern Andes, Left Coast Press, Walnut Creek, USA.

Bender, Barbara (2001) Landscape on-the-move. Journal of Material Culture, 1(1): 75-89.

Bray, Tamara (2015) Andean Wak'as and Alternative Configurations of Persons, Power, and Things. En: The Archaeology of Wak'as: Explorations of the Sacred in the Pre-Columbian Andes, T. L. Bray (ed.) University Press of Colorado, Boulder. Pp. 3-22

Bradley, Bruce y Michael B. Collins (2013) Imagining Clovis as a Cultural Revitalization Movement. En: Paleoamerican Odyssey, Editado por Kelly E. Graf, Caroline V. Ketron, y Michael R. Waters, Texas A\&M University Press, Santa Fe, Texas. Cap 14: 247-255.

Borrero, Luis A. (1990) Evolución cultural divergente en la Patagonia austral. Anales del Instituto de la Patagonia (Serie Ciencias Sociales) 19: 133-139.

Borrero, Luis A. (2005) The Archaeology of the
Patagonian Deserts Hunter-Gatherers in a Cold Desert. En: Desert Peoples, Archaeological Perspectives, editado por P. Veth, M. Smith, P. Hiscock, Blackwell Publishing, New York, p. 142-158.

Cornero, S. y W. Neves (2011) Ocupaciones tempranas en las Sierras Centrales: la Gruta de Candonga, Córdoba, Argentina, aportes a su antigüedad. Actas de Resúmenes del $1^{\circ}$ Congreso Internacional de Arqueología de la Cuenca del Plata, pp. 218-19. Ciudad Autónoma de Buenos Aires

Fabra, Mariana (2014) Historia de las poblaciones prehispánicas del sector austral de las Sierras Pampeanas: variabilidad morfológica y modelos arqueológicos. Editorial de la FFyH, Facultad de Filosofía y Humanidades, Universidad Nacional de Córdoba, Córdoba

Fabra, Mariana, Andrés Laguens, Darío Demarchi (2005) Análisis intra e interpoblacional de rasgos craneanos no métricos en aborígenes prehispánicos del actual territorio de Córdoba. En: Revista Argentina de Antropología Biológica 7(2): 47-65.

Fabra, Mariana, Andrés Laguens, Darío Demarchi (2007) Human colonization of the central territory of Argentina: design matrix models and craniometric evidence. American Journal of Physical Antrhopology, 133 (4): 1060-1066.

Flegenheimer, Nora; Laura Miotti y Natalia Mazzia (2013) Rethinking Early Objects and Landscapes in the Southern Cone: Fishtail-Point Concentrations in the Pampas and Northern Patagonia. En: Paleoamerican Odyssey, Editado por Kelly E. Graf, Caroline V. Ketron, y Michael R. Waters, Texas A\&M University Press, Santa Fe, Texas. Cap. 21: $359-376$.

Gibson, James J. (1986) Theory of affordances. En: The ecological approach to visual perception, DE Gibson, J. J., Hillsdale, NJ: Erlbaum, cap. 8: 127-143.

Hallowell, A. Irving (1964) [1960] Ojibwa Ontology, Behavior and World View. En: Primitive Views of the World, Stanley Diamond (ed.), pp. 49-82. New York: Columbia University Press.

Howell, Signe (1989) 'To Be Angry Is Not To Be Human, But To Be Fearful Is': Chewong concepts of human nature. En: From Societies at Peace: Anthropological Perspectives, editado por Signe Howell y Roy Willis, London and New York: Routledge, p. 45-59.

Ingold, Tim (2007) Materials against materiality. Archaeological Dialogues 14 (1): 1-16

Kelly, Robert L. (2003) Colonization of new land by hunter-gatherers. Expectations and implications based 
on ethnographic data. En: Colonization of Unfamiliar Landscapes. The Archaeology of Adaptation, editado por M. Rockman y J. Steele, Routledge, Londres, Cap. 3: 44-58.

Kohn, Eduardo (2013) How forest think. Toward an anthropology beyond the human. University of California Press, Berkeley and Los Angeles, California

Laguens, Andrés, Darío Demarchi y Mariana Fabra (2007) Un estudio arqueológico y bioantropológico de la colonización humana en el sector sur de las Sierras Pampeanas. Debates actuales en Arqueología y Etnohistoria, E. Olmedo and F. Ribero (comp.), pp. 119134, Universidad Nacional de Rio Cuarto.

Laguens, Andrés, Darío A. Demarchi y G. Roxana Cattáneo (2007a) Estancia La Suiza: una localidad arqueológica en relación al poblamiento inicial de las Sierras Centrales Resúmenes ampliados del XVI Congreso Nacional de Arqueología Argentina. Revista Pacarina III: 471-475. Jujuy.

Laguens, A., E. Pautassi, G. Sario, y R. Cattáneo (2007b) ELS1, a Fishtail Projectile-Point Site from Central Argentina. Current Research in the Pleistocene 24:5557.

Laguens, Andrés, Darío Demarchi, Mariana Fabra (2007c) Un estudio arqueológico y bioantropológico de la colonización humana en el sector sur de las Sierras Pampeanas. Debates actuales en Arqueología y Etnohistoria, E. Olmedo and F. Ribero (comp.), pp. 119134, Universidad Nacional de Rio Cuarto.

Laguens, Andrés, Roxana Cattáneo, Eduardo Pautassi y Gisela Sario (2009) Poblamiento humano temprano en las Sierras de San Luis: Estancia La Suiza. En: Las sociedades de los paisajes áridos y semi-áridos del Centro-Oeste argentino, ed. por Y.Martini, G. Pérez Zavala y Y. Aguilar, Editorial UN de Río Cuarto, pp. 4160.

Laguens, Andrés (2009) De la diáspora al laberinto. Notas y reflexiones sobre la dinámica relacional del poblamiento humano en el centrosur de Suramérica. Arqueología Suramericana 5(1):42-67.

Leroi-Gourhan, André (1965) Le geste et la parole. Vol. 2 : La mémoire et les rythmes. Paris, Albin Michel.

Lima, Tânia Stolze (1999) The Two and its Many: Reflections on Perspectivism in a Tupi Cosmology. Ethnos 64(1):107-131.

Lima, Tânia Stolze (2000) Towards an Ethnographic Theory of The Nature/Culture Distinction in Juruna Cosmology . Brazilian Review of Social Sciences, special issue, no. 1: 43-52
Meltzer, David J. (2013) The human colonization of the Americas: archaeology. En: The Encyclopedia of Global Human Migration, editado por Immanuel Ness, Blackwell Publishing Ltd, Londres. Cap. 8: 1-9.

Miotti, Laura L. y Mónica C. Saleme (2003) When Patagonia was colonized: people mobility at high latitudes during Pleistocene/Holocene transition. Quaternary International, 109-110: 95-111.

Mora, Santiago (2006) Amazonia, pasado y presente de un territorio remoto. Bogotá: Universidad de los AndesFondo de Promoción a la Cultura

Nores, Rodrigo y Darío A. Demarchi (2011) Análisis de haplogrupos mitocondriales en restos humanos prehispánicos del área central de Argentina. Revista Argentina de Antropología Biológica 13:43-54.

Nores, Rodrigo, Mariana Fabra y Darío A.Demarchi (2011) Variación temporal y espacial en poblaciones prehispánicas de Córdoba. Análisis de ADN antiguo. Revista del Museo de Antropología 4:187-194. Facultad de Filosofía y Humanidades, UNC,Córdoba.

Patané Aráoz, Claudio J. y Nami, Hugo G. (2014) The First Paleoindian Fishtail Point Find in Salta Province, Northwestern Argentina. Archaeological Discovery, 2, 26-30. http://dx.doi.org/10.4236/ad.2014.22004

Pedersen, M.A. (2007) Talismans of thought: shamanist ontologies and extended cognition in northern Mongolia, En: A. Henare, M. Holbraad and S. Wastell, eds., ThinkingThrough Things: Theorising Artefacts Ethnographically. London: Routledge.

Politis, Gustavo G., Pablo G. Messineo y Cristian A. Kaufmann (2004) El poblamiento temprano de las Ilanuras pampeanas de Argentina y Uruguay. Complutum, Vol. 15: 207-224.

Rivero, Diego y Eduardo Berberián (2011) Paleoindian Occupation in the Central Mountains of Argentina: Was It a Failed Colonization? Current Research in the Pleistocene, 28: 118-120

Rivero, Diego y Fabiana Roldán (2005) Initial Peopling of the Córdoba Mountains, Argentina: First Evidence from El Alto 3. Current Research in the Pleistocene 22:33-35.

Rockman, Marcy y James Steele (2003) Colonization of Unfamiliar Landscapes. The Archaeology of Adaptation. M. Rockman y J. Steele (editores), Routledge, London.

Salomon, Frank (1991) Introductory essay: The Huarochiri Manuscript. In: The Huarochiri Manuscript; A Testament of Ancient and Colonial Religion, F. Salomon and G. L. Urioste, eds., Austin, University of Texas Press. pp. 1-38. 
Sario, Gisela (2010) El aprovisionamiento de las rocas. Un caso de estudio en la localidad arqueológica de Estancia La Suiza. Actas del XVII Congreso Nacional de Arqueología Argentina. Tomo V: 1741-1745. Mendoza.

Sario, Gisela y Eduardo Pautassi (2010) El aprovisionamiento de las rocas. Un caso de estudio en la localidad arqueológica de Estancia La Suiza. Actas del XVII Congreso Nacional de Arqueología Argentina. Tomo V: 1741-1745. Mendoza.

Troncoso, Andrés (2014) Relacionalidad, prácticas, ontologías y arte rupestre en el centro norte de Chile (2000 a.C. a 1.540 d.C.). Revista de Arqueologia , 27(2): 64-87.

Vilaça, Aparecida (2009) Bodies in perspective: a critique of the embodiment paradigm from the point of view of Amazonian ethnography.In: Social Bodies, H. Lambert and M. McDonald (eds.), Oxford: Berghahn Books: 129-147

Viveiros de Castro, Eduardo (1998) Cosmological Deixis and Amerindian Perspectivism. Journal ofthe Royal Anthropological Institute 4(3) 469-88.

Viveiros de Castro, Eduardo (2004a) Exchanging perspectives. The transformation of objects into subjects in Amerindian ontologies. Common Knowledge 10 (3): 463-84.

Viveiros de Castro, Eduardo (2004b) Perspectival anthropology and the method of controlled equivocation, Tipití (Journal of the Society for the Anthropology of Lowland South America) 2(1): 3-22.

Webb, R. Esmée y David J. Rindos (1997) The Mode and Tempo of the Initial Human Colonisation of Empty Landmasses: Sahul and the Americas Compared. Archaeological Papers of the American Antrhopological Association, 7(1): 233-250.

Willerslev, Rane (2007) Soul hunters: hunting, animism and personhood among the Siberian Yukaghirs. Berkeley: University of California Press. 